\title{
ROLE OF MECHANICAL VESTIBULAR STIMULATION ON BALANCE IN CHILDREN WITH DOWN SYNDROME
}

Amr A. Ellithy ${ }^{1}$; Elham E.Salem ${ }^{2}$;p Nahla M. Ibrahim ${ }^{3}$ and Ehab R. Abd El Rauof ${ }^{4}$.

${ }^{1}$ Master Degree in Physical Therapy for Pediatrics, Faculty of Physical Therapy, Cairo University, Egypt.

${ }^{2}$ Professor, Department of Physical Therapy for Pediatrics ,Faculty of Physical Therapy ,Cairo University,

${ }^{3}$ Lecture, Department of Physical Therapy for Pediatrics ,Faculty of Physical Therapy ,Cairo University, Egypt.

${ }^{4}$ Professor of Clinical Genetics , Department of Research of Children with Special needs, National Research Center, Egypt.

\section{ABSTRACT:}

Background: Motor and cognitive development of children with Down syndrome (DS) is delayed and inharmonic. Neuro-muscular abnormalities, such as hypotonia, retained primary reflexes, and slow performance of volitional reaction, result in difficulties with body balance. Purpose and Methods: It was to investigate the effect of mechanical vestibular stimulation on balance in children with Down syndrome. Thirty children with Down syndrome participated in this study. Subjects were divided into two groups group A and group B, fifteen children in each group, the control group received a selected physical therapy program designed for those cases and the study group received the same program in addition to mechanical vestibular stimulation. Children were evaluated before and after 3 successive months of treatment program by biodex balance system to evaluate their balance regarding anteroposterior stability index (APSI), mediolateral stability index (MLSI) and overall stability index (OASI). Results: There was a significant decrease in APSI, MLSI and OASI post treatment compared with that pretreatment in the control and study groups $(\mathrm{p}<$ 0.0001). Comparison between both groups post treatment revealed a significant improvement by the decrease of degrees of sway in APSI, MLSI and OASI of the study group compared with that of the control $\operatorname{group}(\mathrm{p}<0.05)$.

Conclusion: Results of the study suggested the use of mechanical vestibular stimulation in addition to the designed program to improve balance in children with down syndrome.

Key Words: Down syndrome - Biodex - balance- mechanical vestibular stimulation 


\section{INTRODUCTION:}

Children with Down syndrome may experience difficulty in maintaining balance due to certain cause as hypotonia, laxity of ligaments, decrease postural control, decrease muscle strength. This is beside the mental retardation so studies state that general at motor skills development has been below than of normal children ${ }^{(\mathbf{1})}$.

The Biodex balance system is a unique dynamic postural control assessment and training system. It is a multi-axial device that objectively measures and records the individual's ability to maintain stability under dynamic stress. It is less expensive, more portable that include movable platform that can be adjusted to provide varying degrees of stability and offer computer-based data ${ }^{(2)}$.

There are different methods that can improve balance in children with down syndrome such as Facilitation of trunk control to improve postural control from different positions (prone, supine, sitting). Balance training was carried from different positions (quadruped, kneeling, half kneeling and standing) on tilting board. Facilitation of righting, protective and equilibrium reactions: These exercises were carried through tilting from different positions (forward, backward, and sideways) in order to improve postural mechanisms via variety of exercises applied on medical ball and tilting board ${ }^{(3)}$.

Sensory aspects components to control balance consist of The Sensory System: Afferent information for postural control system comes from visual, vestibular and somatosensory inputs. Visual Inputs that report information regarding the position and motion of the head with respect to the surrounding objects. They are an important source of information for postural control ${ }^{(4)}$

The Vestibular System provides the Central Nervous System (CNS) with information about the position and movement of the head with respect to gravity, providing a gravitational frame of reference for postural control and Somatosensory Inputs that provide information concerning the orientation of body parts to one another and to the support surface $^{(4)}$.

Vestibular stimulation is used in physical therapy for stimulating normal development of postural control on children with neurodevelopment disabilities. Therapeutic vestibular stimulation increases the brain's capacity to integrate vestibular stimuli by developing motor responses that aid integration. As tolerance in capacity to organize vestibular stimuli increases, children with sensory motor dysfunction begin to seek out motor activities which will stimulate their own Vestibular System. Slow and regular stimulation can be used for hypertonic children but fast and irregular stimulation can be used for hypotonic children ${ }^{(5)}$. 
Relearning of postural control through external visual, auditory and vestibular feedback is believed to be an effective therapy for improving balance control. It is thought that by giving patients additional visual information, they will become more aware of the body's displacements and orientation in space ${ }^{(6)}$. Mechanical vestibular stimulation used in remediation of sensory integrated dysfunction. Stimulation was provided by swinging or spinning a child while he was lying or sitting in a net hammock $^{(7)}$. Fast vestibular stimulation promotes a generalized facilitation of postural tone, postural reaction, motor control and increase arousal and attention. Therapeutically stimulation can be achieved using variety of equipment including tilt board, scooter board or spinning chair. Both linear and angular motion cab be use ${ }^{(8)}$

\section{MATERIAL AND METHODS:}

Ethical approval was obtained from the institutional review board at faculty of physical Therapy, Cairo university before the commence of the study (No: P.T.REC/012/001795).

\section{Subjects:}

Thirty children with Down syndrome had participated in this study. They were selected from the outpatient clinic, faculty of physical therapy, Cairo university. An informed consent was obtained by each parent after explaining the nature, purpose and benefits of the study protocol informing them of their right to refuse or withdraw at any time and about the confidentiality of any obtained information.

\section{Inclusion criteria:}

The children were selected according to the following criteria: Their age ranged from 5 to 10 years. They were able to understand the verbal commands, Their Body Mass Index (BMI) within normal range, their height not less than 1 meter to see the screen of Biodex balance system, they were able to stand momently and they had history of frequent falling during walking.

\section{Exclusion Criteria:}

The children were excluded from this study if they had any of the following criteria: Severe Atlanto Axial instability, Severe mental retardation and uncontrolled cardiopulmonary problems.

Children were assigned into two groups (control (A) and study (B) group) of equal numbers. Their balance was assessed before and after 3 successive months of conduction of the treatment program.

\section{Control Group(A)}

Children in this group had received physical therapy exercise program designed for down syndrome cases that included regular balance exercises for one hour/day, 3 sessions/week for three successive months. 


\section{Study Group(B)}

Children in this group had received the same selected physical therapy exercise program for 45 minutes in addition to mechanical vestibular stimulation (MVS) using swing system for $15 \mathrm{~min}$.

\section{Evaluation:}

\section{1- Weight and Height scale:}

a valid and reliable weight and height scale was used to measure the weight and height of the children of both group prior to the test that used as an entering data to the device included in the test

\section{2-Biodex Balance System:}

The Biodex balance system is a unique dynamic postural control assessment and training system. It was used for assessment of children of both group before and after 3 successive months of treatment

\section{Procedure:}

- The child's weight, height and chronological age were entered to control screen display located in front of the subject.

- Position of the support handle and its height was adjusted according to child height which the child asked to grasp it during initiation of the test and leave it as the test proceeds.

- Each child was tested without footwear to achieve full contact with platform and was asked to perform two trials before recording to be familiar with the device. Each child was asked to stand on both feet with arms held at sides and trying to maintain balance as much as possible.

- The start key was pressed in control panel to unlock platform with auditory alarm just before the beginning of the test.

- Each child was asked to maintain balance as much as possible because the platform was unstable just after the alarm.

- Three stability indices were obtained before and after treatment program for each subject of the study group including: Anteroposterior stability, Medio-lateral stability index and overall stability index. Three trials were done then average was taken.

\section{Intervention: For control group:}

Each child had received a selected physical therapy exercise program designed for children with down syndrome that directed towards improving both static, dynamic balance and gait as follows:

Strengthening exercise to abdomen \&back, Stability training, facilitation of different postural reactions which is essentials component of postural control, changing positions, Righting and equilibrium reactions and gait training activities.

\section{For study group:}

Each child in this group received the previous selected physical therapy program in addition to Mechanical Vestibular Stimulation. 


\section{Mechanical vestibular stimulation system/ Swing system: Procedures of mechanical vestibular stimulation:}

The child was placed in sitting on the platform swing and his hands grasping ropes then the therapist stood behind him and pushed the platform in fast arrhythmic and jerky movement in back and front for five minutes, side to side for five minutes and in orbital direction for five minutes with the child trying to maintain his balance.

The child was placed in sitting on the disk swing and his legs and arms wrapped around the disk swing then the therapist stood behind him and pushed the platform in fast arrhythmic and jerky movement in back and front for five minutes, side to side for five minutes and in orbital direction for five minutes with the child trying to maintain his balance.

\section{Statistical analysis}

Descriptive statistics and unpaired t-test were conducted for comparison of subject characteristics between both groups. Chi- squared was carried out for comparison of sex distribution between groups. Unpaired t-test was conducted to compare the mean values of APSI, MLSI and OASI between the control and study groups. Paired t-test was conducted for comparison between pre and post treatment in each group. The level of significance for all statistical tests was set at $\mathrm{p}<0.05$. All statistical analysis was conducted through the statistical package for social studies (SPSS) version 25 for windows (IBM SPSS, Chicago, IL, USA).

\section{-Subject characteristics:}

\section{RESULTS}

Table (1) showed the subject characteristics of the control and study groups. There was no significant difference between both groups in age, weight, height and BMI $(\mathrm{p}>0.05)$.Also, there was no significant difference in sex distribution between groups $(p>0.05)$.

Table 1. Basic characteristics of participants.

\begin{tabular}{|c|c|c|c|}
\hline & Control group & Study group & p-value \\
\hline Age, mean \pm (SD), years & $8.26 \pm 1.16$ & $8.33 \pm 1.11$ & 0.87 \\
\hline Weight, mean \pm (SD), kg & $23.33 \pm 1.34$ & $23.6 \pm 1.95$ & 0.66 \\
\hline Height, mean \pm (SD), cm & $123.73 \pm 3.36$ & $123.06 \pm 4.35$ & 0.64 \\
\hline BMI, mean $\pm(\mathrm{SD}), \mathrm{kg} / \mathrm{m}^{2}$ & $15.3 \pm 0.53$ & $15.68 \pm 1.22$ & 0.27 \\
\hline $\begin{array}{l}\text { Sex, n (\%) } \\
\text { Girls }\end{array}$ & $3(20 \%)$ & $6(40 \%)$ & \\
\hline Boys & $12(80 \%)$ & $9(60 \%)$ & 0.23 \\
\hline
\end{tabular}

SD, standard deviation; p-value, level of significance

\section{Effect of treatment on APSI, MLSI and OASI: \\ - Within group comparison:}

There was a significant decrease in APSI, MLSI and OASI post treatment compared with that pretreatment in the control and study groups(p $<0.0001)$. The percent of decrease in APSI, MLSI and OASI in the control 
group were $6.25,10.05$ and $7.94 \%$ respectively, while that in the study group were $23.61,24.42$ and $26.43 \%$ respectively. (Table 2 )

\section{- Between groups comparison:}

There was no significant difference in APSI, MLSI and OASI between both groups pre-treatment $(\mathrm{p}>0.05)$. Comparison between both groups post treatment revealed a significant decrease in APSI, MLSI and OASI of the study group compared with that of the control group $(\mathrm{p}<0.05)$. (Table 2$)$.

Table 2. Mean APSI, MLSI and OASI pre and post treatment of the control and study groups:

\begin{tabular}{|c|c|c|c|c|c|}
\hline \multirow[b]{2}{*}{ APSI } & $\begin{array}{c}\text { Control group } \\
\overline{\mathbf{x}} \pm \text { SD }\end{array}$ & $\begin{array}{c}\text { Study group } \\
\overline{\mathbf{x}} \pm \text { SD }\end{array}$ & MD & t- value & p value \\
\hline & & & & & \\
\hline Pre treatment & $3.52 \pm 0.65$ & $3.6 \pm 0.58$ & -0.08 & -0.32 & 0.74 \\
\hline Post treatment & $3.3 \pm 0.67$ & $2.75 \pm 0.62$ & 0.55 & 2.33 & 0.02 \\
\hline MD & 0.22 & 0.85 & & & \\
\hline$\%$ of change & $6.25 \%$ & $23.61 \%$ & & & \\
\hline \multirow[t]{2}{*}{ t- value } & 7.05 & 12.54 & & & \\
\hline & $p=0.0001$ & $p=0.0001$ & & & \\
\hline \multicolumn{6}{|l|}{ MLSI } \\
\hline Pre treatment & $1.89 \pm 0.52$ & $1.72 \pm 0.41$ & 0.17 & 0.96 & 0.34 \\
\hline Post treatment & $1.7 \pm 0.53$ & $1.3 \pm 0.2$ & 0.4 & 2.72 & 0.01 \\
\hline MD & 0.19 & 0.42 & & & \\
\hline$\%$ of change & $10.05 \%$ & $24.42 \%$ & & & \\
\hline \multirow{2}{*}{ t- value } & 5.39 & 5.84 & & & \\
\hline & $p=0.0001$ & $p=0.0001$ & & & \\
\hline \multicolumn{6}{|l|}{ OASI } \\
\hline Pre treatment & $3.78 \pm 0.49$ & $3.67 \pm 0.52$ & 0.11 & 0.6 & 0.54 \\
\hline Post treatment & $3.48 \pm 0.54$ & $2.7 \pm 0.5$ & 0.78 & 4.19 & 0.0001 \\
\hline MD & 0.3 & 0.97 & & & \\
\hline$\%$ of change & $7.94 \%$ & $26.43 \%$ & & & \\
\hline t- value & 8.52 & 8.19 & & & \\
\hline & $p=0.0001$ & $p=0.001$ & & & \\
\hline
\end{tabular}

$\overline{\mathbf{x}}$, mean; SD, standard deviation; MD, mean difference; $p$-value, probability value

\section{DISCUSSION:}

Thirty children with Down syndrome participated in this study. Subjects were divided into two groups, fifteen children in each group, the control group received a selected physical therapy program and the study group received the selected physical therapy program and mechanical vestibular stimulation. Data obtained from both groups regarding anteroposterior stability index (APSI), mediolateral stability index (MLSI) and overall stability index (OASI) were statistically analyzed and compared. Comparing the general characteristics of the subjects of both groups revealed that there was no significance difference between the two groups in the mean age, weight, height and BMI ( $p>0.05)$.

Concerning the pre and post treatment results of the measured variables (overall SI,antero-posterior SI and medio-lateral SI) of down syndrome 
children of both groups. the results have significant improvement in both groups. However, more improvement with significant difference was noticed in favor of the study group. concern the control group has significant improvement in the post treatment mean values of the measured variables after receiving the traditional physical therapy treatment program which confirm the importance of physical therapy treatment of children with down syndrome specially when concern with improving standing and walking abilities. improvement in post treatment mean values of both groups may be attributed to increase of activity of antigravity muscle which counteract the force of gravity and leads to modulation of postural tone. the improvement in the study group might be due to the mechanical vestibular stimulation program which comes on the same line with findings of ${ }^{(9)}$ who found that, proprioceptive awareness of postures and movement is most required during the learning of new skills. The post treatment improvements of the study group may be due to involvement of the stimulation of many systems and sub systems as nervous, muscular, vestibular, visual and proprioceptive system during vestibular stimulation part of sessions. this supported by Brooks ${ }^{(10)}$ who suggested that postural control is the result of many systems working together in a goal directed situation. It can be suggested that integration of mechanical vestibular stimulation program with the designed physical therapy program has a significant effect on balance in children with down syndrome, so it should be considered as an important therapeutic modality for treatment of these cases and adding mechanical vestibular stimulation to the treatment sessions of down syndrome is highly recommended.

\section{REFERENCES}

1) Cynthia, H. and S.E. Pamela (2001): How man moves: Kinesiological studies and methods. William Heinemann, Londan. $3^{\text {rd }}$ Edition, pp: 210-215.

2) Hinman, M. (2000): "Factors affecting reliability of the biodex balance system: a summary of four studies". Sport Rehab.,9:240-252.

3) Evans- Martin, F.(2009): Genes and disease Down syndrome, Chelsea House, New York, 68-88.

4) Cook, A.S. and M.H. Woollacott (2001): Neural and musculoskeletal contributions to the development of stance balance control in typical children with cerebral palsy. Acta Pediatric suppl., 416:58-62.

5) Helt, D.G. and R. Foulds (2004): Vestibular stimulation and its effect on muscle tone. Bio-engineering Conference, Volume, Issue, 17-18 April pages: 7-8. 
6) Peppan, V. ; M. Kortsmit ; E. Lindeman and G. Kwakkel (2006); Effect of visual feedback therapy on postural control in bilateral standing after stroke: a systemic review. J Rehabil. Med; 38-39.

7) Ayres, J.A. (1979): Sensory integration and learning disorders. Western psychological services, Los Angelos, pp: 127-138).

8) Heinger, M. and S. Randolph (1981): Neurophysiological stimulation and its effects on muscle tone .Bioengineering conference . (17-18):7-8.

9) Tristan, D. and R. Martin (2004): Understanding Balance; The mechanics of posture and locomotion, Nelson Throne :601-605.

10) Brooks, V.B.(2003): The neural basis of motor control, oxford University Press, new York,pp:5 :129-150.

11) Gray, H.(2004): Elevating "the gravity" from gravity. Gray's anatomy. 29th ed.

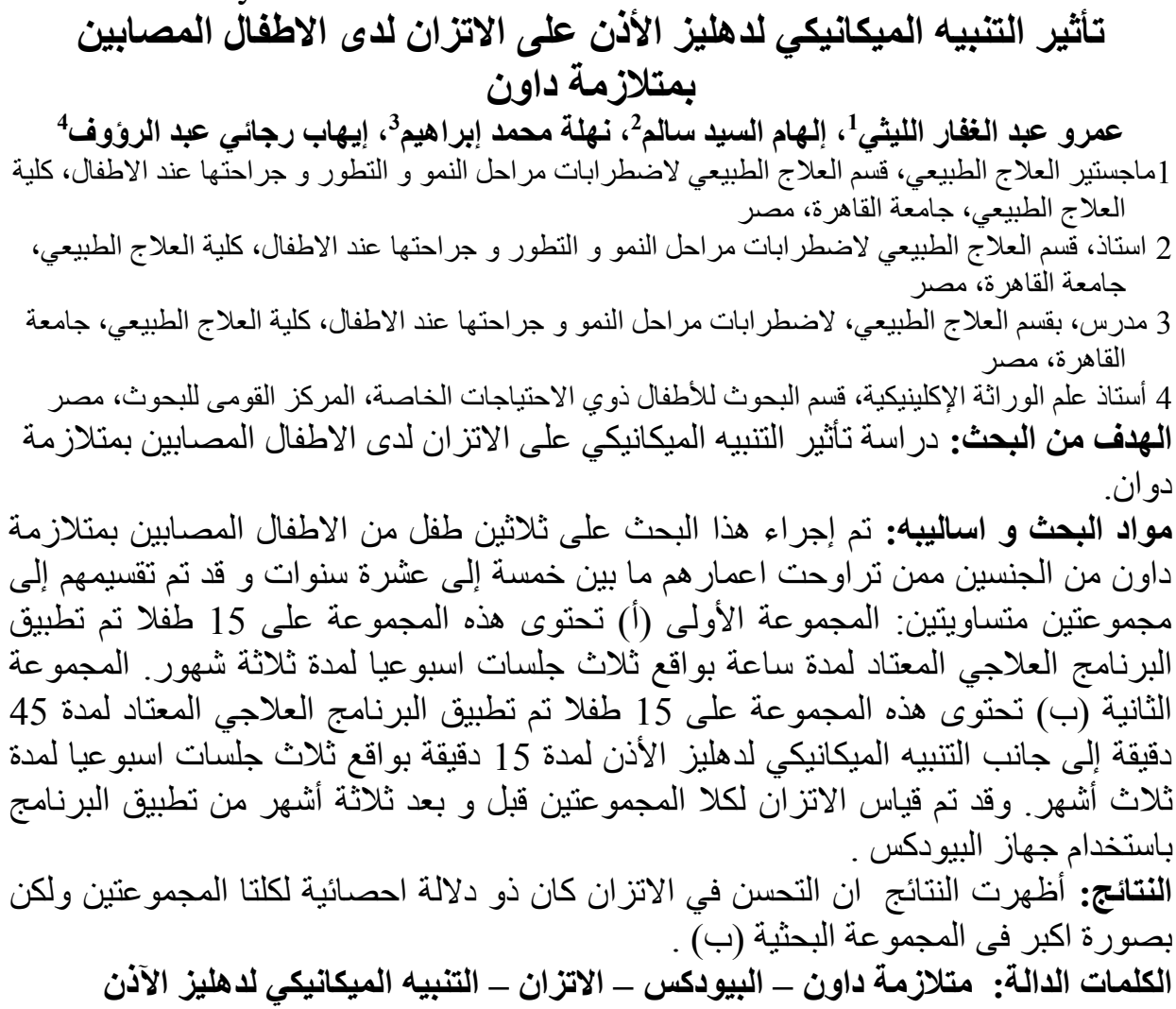

